\title{
Hybrid Virtual Holonomic Constraints for a 2-D.O.F. Bipedal Robot
}

\author{
Mohamed Al Lawati and Christopher Nielsen
}

\begin{abstract}
We apply virtual holonomic constraints (VHCs) to a fully actuated Euler-Lagrange system with impacts : a bipedal walking robot consisting of a stance leg, swing leg and hip mass. We call these hybrid virtual holonomic constraints (hVHCs). For any desired gait of the bipedal robot, the angular position of the swing leg is expressed as a function of the angular position of the stance leg. Using this function, a hVHC is formed and the control objective is to constrain the dynamics of the robot to evolve on the constraint manifold. A design procedure is developed to generate feasible hVHCs for a 2-DOF bipedal robot. Simulation results are presented.
\end{abstract}

\section{INTRODUCTION}

Virtual holonomic constraints (VHC) have been widely used and applied to continuous-time Euler-Lagrange systems. Shiriaev et al. [1] used the notion of VHC to find an integral of motion of the so called reduced system, or zero dynamics, of a class of $n$-DOF mechanical systems subject to $n-1$ VHCs. This integral of motion is helpful in understanding the dynamics of the reduced system. It has been also used to evaluate gait stability of a three-link bipedal robot by computing transverse coordinates [2]. A similar integral of motion was developed by Maggiore and Consolini [3].

The notion of VHC has also been used to generate gaits of bipedal robots. Among the advantages of VHC approach for gait generation is that it does not require pre-computed trajectories as in the case of $\mathrm{ZMP}^{1}$-based controllers [4]. In addition, utilizing VHCs makes it easy to achieve different gaits by modifying the constraint function.

$\mathrm{Hu}$ et al. [5] utilize VHCs to design controllers that balance fully actuated planar bipedal robots with feet during disturbed standing. Under small disturbances, their controller balances the robot using the support from a single leg.

Grizzle and his collaborators have studied the zero dynamics of under-actuated planar bipedal robots [6], [7] and [8]. The results in [9] use control Lyapunov functions to stabilize the zero dynamics manifold of a bipedal robot exponentially fast. To keep the constraint manifold invariant under impacts, the work of [8] and [9] define a set of output functions using Bézier polynomials. They use the intrinsic features of Bézier polynomials to develop conditions leading to impact invariance. Experimental validation of virtual constraintsbased control, including running and walking on uneven

This work was partially supported by the Natural Sciences and Engineering Research Council of Canada (NSERC) and by Sultan Qaboos University.

M. Al Lawati is with the Department of Mechanical and Industrial Engineering, Sultan Qaboos University, Muscat, Oman. mlawati@squ.edu.om

C. Nielsen is with the Department of Electrical and Computer Engineering, University of Waterloo, Ontario, Canada. cnielsen@uwaterloo.ca

${ }^{1}$ Zero Moment Point. terrain, has also been extensively performed [10], [11], [12] and [13].

In this paper we provide conditions (Section IV) guaranteeing impact invariance for a fully actuated Euler-Lagrange system with impacts for arbitrary, possibly non-polynomial, constraints. For a 2-DOF bipedal robot we provide a systematic design procedure using constant coefficient polynomials that returns a valid hVHCs. The polynomials allow the designer to design a large family of gait shapes (Section IVA). We design two gait controllers using hVHCs. Both controllers enforce a given gait shape modelled as a hVHC. One feedback controls the dynamics on the constraint manifold while the other leaves the constraint manifold dynamics uncontrolled.

Among other attempts in gait control for bipedal robots, Yan et al. [14] have added a locking mechanism at the hip of an under-actuated 2-DOF bipedal robot that locks the swing leg when it retracts. This results in two impact events within a single gait cycle; one corresponds to locking and the other to ground collision. In order to stabilize a gait, they use the method of transverse coordinate transformation to approximate their model by a linear impulsive system. This approach was also taken by [15] and [2]. Other notable gait generation strategies based on mechanical energy considerations include [16] and [17].

The approach taken here is to view a hybrid limit cycle in the state-space of a 2-DOF bipedal robot as a constraint imposed between the two legs: stance and swing. The functional constraint defines a subset of the robot's state space which we call the constraint manifold. When the system is restricted to evolve on the constraint manifold, the robot follows the desired gait. The control design problem then reduces to the problem of stabilizing the constraint manifold. We achieve this objective using feedback linearization, similar to the work [7], [8].

\section{General Problem Formulation}

Consider an Euler-Lagrange system with an $N$ dimensional configuration space $\Theta \subseteq \mathbb{R}^{N}$ and $N$ control inputs $\tau \in \mathbb{R}^{N}$. The model can be written in the standard vector form

$$
M(\theta) \ddot{\theta}+C(\theta, \dot{\theta}) \dot{\theta}+G(\theta)=B(\theta) \tau
$$

where $\theta \in \Theta, M(\theta) \in \mathbb{R}^{N \times N}$ is the inertia matrix, $C(\theta, \dot{\theta}) \in \mathbb{R}^{N \times N}$ represents the centripetal and Coriolis terms and $G(\theta) \in \mathbb{R}^{N}$ represents the gravitation effects. The systems and $B(\theta) \in \mathbb{R}^{N \times N}$ is non-singular everywhere. System (1) models a large class of physical systems including the bipedal robot studied in this paper. 
When a bipedal robot's feet hit the ground, the dynamical model (1) undergoes an instantaneous change in its state $(\theta, \dot{\theta}) \in \mathbb{R}^{N} \times \mathbb{R}^{N}$. This observation motivates us to define an "impact surface" $\mathcal{S} \subset \mathbb{R}^{N} \times \mathbb{R}^{N}$. The impact surface has the property that when $(\theta, \dot{\theta}) \in \mathcal{S}$, the state instantaneously changes to $\left(\theta^{+}, \dot{\theta}^{+}\right)=J(\theta, \dot{\theta})$ where $J: \mathbb{R}^{N} \times \mathbb{R}^{N} \rightarrow$ $\mathbb{R}^{N} \times \mathbb{R}^{N}$ is smooth and $J(S) \cap S=\varnothing$.

These considerations lead to a special class of hybrid Euler-Lagrange systems, denoted $\mathrm{EL}_{\mathcal{H}}$, given by

$$
\mathrm{EL}_{\mathcal{H}}: \begin{cases}M(\theta) \ddot{\theta}+C(\theta, \dot{\theta}) \dot{\theta}+G(\theta)=B(\theta) \tau, & (\theta, \dot{\theta}) \notin \mathcal{S} \\ \left(\theta^{+}, \dot{\theta}^{+}\right)=J(\theta, \dot{\theta}), & (\theta, \dot{\theta}) \in \mathcal{S} .\end{cases}
$$

We recall the definition of virtual holonomic constraints for Euler-Lagrange systems from [3].

Definition II.1 ([3]). A virtual holonomic constraint (VHC) of order $k$ for System (1) is a relation $h(\theta)=0$ where $h: \Theta \rightarrow \mathbb{R}^{k}$ is smooth, $\operatorname{rank}\left(\mathrm{d} h_{\theta}\right)=k$ for all $\theta \in h^{-1}(0)$, and the set

$$
\Gamma:=\left\{(\theta, \dot{\theta}): h(\theta)=\mathrm{d} h_{\theta} \dot{\theta}=0\right\}
$$

is controlled invariant. That is, there exists a smooth feedback $\tau(\theta, \dot{\theta})$ such that $\Gamma$ is positively invariant for the closedloop system. The set $\Gamma$ is called the constraint manifold associated with the VHC $h(\theta)=0$. A VHC is stabilizable if there exists a smooth feedback $\tau(\theta, \dot{\theta})$ that asymptotically stabilizes $\Gamma$. In this case $\tau(\theta, \dot{\theta})$ is said to enforce the VHC $h(\theta)=0$.

Following [3] it is convenient to adopt a parametric description of the $\mathrm{VHC}$ in which the first $k$ configuration variables are expressed as smooth functions of the remaining $N-k$ configuration variables

$$
\theta_{i}=p_{i}\left(\theta_{k+1}, \ldots, \theta_{N}\right), i \in\{1, \ldots, k\} .
$$

Here, $h(\theta)=\left(\theta_{1}-p_{1}(\theta), \ldots, \theta_{k}-p_{k}(\theta)\right)$. We are now ready to state the general problem.

Hybrid VHC enforcement problem : Given a hybrid EulerLagrange system (2) and a virtual holonomic constraint of order $k$, find if possible, a feedback control law $\tau(\theta, \dot{\theta})$ such that,

- The feedback enforces the VHC for the continuous-time dynamics in (2) in the sense of Definition II.1.

- The constraint manifold is invariant under the discretetime dynamics in (2), i.e.,

$$
J(\mathcal{S} \cap \Gamma) \subseteq \Gamma .
$$

- The dynamics (2) restricted to $\Gamma$ satisfy application specific constraints such as boundedness, no finiteescape time, trajectory tracking, etc.

The above problem is quite general. In this paper we study a specialized version of the problem applied to a simple compass bipedal walker. We show in Section III, the bipedal walker is a system of the form (2) with $N=2$ and where the impact surface $\mathcal{S}$ is determined by the terrain on which the bipedal robot is walking on. We will consider VHC of order 1 parametrized as in (4). In this case, the function $p_{1}: \mathbb{R} \rightarrow \mathbb{R}$ defines the VHC $h(\theta)=\theta_{1}-p_{1}\left(\theta_{2}\right)$. We impose conditions on $p_{1}$ that guarantee $h$ is a hVHC.

\section{THE MODEL}

Due to the impact events of a walking 2-DOF bipedal robot, the model has a dual dynamical behavior: continuoustime and discrete-time. To model a 2-DOF bipedal robot, different choices of coordinate system are found in the literature. In this paper, we adapt the coordinates shown in Fig. 1.

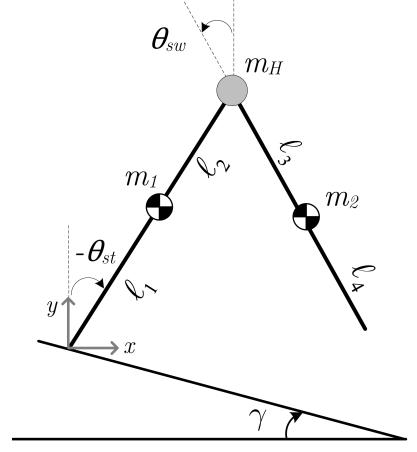

Fig. 1: The model of the 2-DOF bipedal robot.

\section{A. Continuous-time Dynamics}

Let $\theta:=\left(\theta_{\mathrm{st}}, \theta_{\mathrm{sw}}\right)$, the 2-DOF bipedal robot of Fig. 1 is written in the form (1), where the details of matrices $M(\theta)$, $C(\theta, \dot{\theta}), G(\theta)$ and $B(\theta)$ are given in [18].

\section{B. Discrete-time Dynamics}

The discrete-time dynamics consist of two entities:

i. The "impact surface", which in the case of the 2-DOF bipedal robot is the set where the swing foot reaches the ground surface from above followed by an impact event, and

ii. The "reset map", which represents the instantaneous change in positions and velocities. The reset map is derived by redefining the coordinates and using the principle of conservation of angular momentum.

The impact surface is ${ }^{2}$

$$
\begin{gathered}
\mathcal{S}=\left\{(\theta, \dot{\theta}) \in \Theta: H(\theta)=0 \text { and } \mathrm{d}_{\theta} H(\theta) \dot{\theta}<0\right\} \backslash \\
\left\{(\theta, \dot{\theta}) \in \mathbb{R}^{4}: \theta_{\mathrm{st}}=\theta_{\mathrm{sw}}\right\}
\end{gathered}
$$

where the height function, $H(\theta)$, is

$$
H(\theta):=\cos \left(\theta_{\mathrm{st}}+\gamma\right)-\cos \left(\theta_{\mathrm{sw}}+\gamma\right)
$$

with $\gamma \in[0, \pi / 2)$ defined as in Figure 1 . The impact surface (6) excludes the mid-stance point $\theta_{\text {st }}-\theta_{\mathrm{sw}}=0$. The reset map depends on ground impact events, which we assume are (i) impulsive, (ii) inelastic, (iii) instantaneous, and

\footnotetext{
${ }^{2} \mathrm{~A}$ similar impact surface can be found in [19].
} 
(iv) do not cause slipping. Hereafter, we use the superscript "-" to denote variables just before impact and the superscript "+" to denote variables just after impact.

Impact instantaneously affects the configuration variables by switching the role of stance and swing legs $\theta_{\mathrm{st}}^{+}=\theta_{\mathrm{sw}}^{-}$, $\theta_{\mathrm{sw}}^{+}=\theta_{s t}^{-}$. Under assumptions (i)-(iv), angular velocities just after impact are evaluated using conservation of angular momentum at the impact instant. Angular momentum is conserved about [20] (i) the impact point for the whole mechanism, and (ii) the hip mass point for the former stance leg. As a result, the overall reset map can be written as

$$
\left[\begin{array}{c}
\theta^{+} \\
\dot{\theta}^{+}
\end{array}\right]=J\left(\theta^{-}, \dot{\theta}^{-}\right)=\mathbf{J}\left(\theta^{-}\right)\left[\begin{array}{c}
\theta^{-} \\
\dot{\theta}^{-}
\end{array}\right]
$$

where

$$
\mathbf{J}\left(\theta^{-}\right):=\left[\begin{array}{cccc}
0 & 1 & 0 & 0 \\
1 & 0 & 0 & 0 \\
0 & 0 & \hat{\mathbf{J}} \\
0 & 0 &
\end{array}\right], \hat{\mathbf{J}}=\left[\begin{array}{ll}
J_{11}^{b} & J_{12}^{b} \\
J_{21}^{b} & J_{22}^{b}
\end{array}\right]^{-1}\left[\begin{array}{ll}
J_{11}^{a} & J_{12}^{a} \\
J_{21}^{a} & J_{22}^{a}
\end{array}\right]
$$

The expressions of $J_{i j}^{a}$ and $J_{i j}^{b}, i, j \in\{1,2\}$ are given in [18].

\section{The Hybrid Model}

Let $x:=\left[\begin{array}{llll}\theta_{\mathrm{st}}, & \theta_{\mathrm{sw}}, & \dot{\theta}_{\mathrm{st}}, & \dot{\theta}_{\mathrm{sw}}\end{array}\right]^{\top} \in \mathbb{R}^{4}$ define the state vector of the robot. Then, the continuous-time dynamics of the 2-DOF bipedal robot can be written in state-space form as a control-affine system $\dot{x}=f(x)+g(x) \tau$ with input $\tau \in \mathbb{R}^{2}$. Here $f: \mathbb{R}^{4} \rightarrow \mathbb{R}^{4}$ and $g: \mathbb{R} \rightarrow \mathbb{R}^{4 \times 2}$ can be directly evaluated from Equation (1) with appropriate matrices. Following the framework of [21], the robot can be modelled as a hybrid automaton, $\mathcal{H}$, with the following entities,

- The set of discrete states, $\mathcal{Q}=\left\{q_{1}\right\}$, is a singleton since the system has one mode.

- The domain map Domain : $\mathcal{Q} \rightrightarrows \mathbb{R}^{4}$ is defined as Domain $\left(q_{1}\right)=\mathbb{R}^{4}$.

- The flow map $F: \mathcal{Q} \times \mathbb{R}^{4} \rightarrow \mathbb{R}^{4}$ describes a differential equation that defines the continuous-time evolution of the continuous state variable. The flow map is defined as $F\left(q_{1}, x\right)=f(x)+g(x) \tau$.

- Since there is only one mode, there is only one edge and therefore Edges $=\left\{\left(q_{1}, q_{1}\right)\right\}$.

- The guard map Guard : Edges $\rightrightarrows \mathbb{R}^{4}$ is defined as $\operatorname{Guard}\left(q_{1}, q_{1}\right)=\mathcal{S}$ where $\mathcal{S}$ is given by (6).

- The reset map Reset : Edges $\times \mathbb{R}^{4} \rightarrow \mathbb{R}^{4}$ equals $\operatorname{Reset}\left(q_{1}, q_{1}, x\right)=J(x)$.

To represent a 2-DOF bipedal robot compactly, the above data can be summarized by

$$
\mathrm{EL}_{\mathcal{H}}: \quad \begin{cases}\dot{x}=f(x)+g(x) \tau, & x \notin \mathcal{S} \\ x^{+}=J(x), & x \in \mathcal{S} .\end{cases}
$$

\section{HYBRID VHC}

We begin by modifying Definition II.1 for the purposes of this paper.
Definition IV.1. A hybrid virtual holonomic constraint of order $k$ ( $h V H C$ ) for system (2) is a continuous-time VHC of order $k$ such that the constraint manifold is invariant under the discrete-time dynamics

$$
J(\mathcal{S} \cap \Gamma) \subseteq \Gamma .
$$

Based on Definition IV.1, a hVHC can be satisfied even if the states "jump" instantaneously due to discrete-time dynamics.

Assumption 1. The VHCs for system (2) considered in this paper are of order $N-1$ and have the form

$$
h\left(x_{1}, \cdots, x_{N}\right)=\left(x_{2}-p_{1}\left(x_{1}\right), \cdots, x_{N}-p_{N-1}\left(x_{1}\right)\right)
$$

where $p_{i}: \mathbb{R} \mapsto \mathbb{R}$ are $C^{2}, i \in\{1,2, \cdots, N-1\}$.

The next result proposes conditions on a VHC of degree $N-1$ for continuous dynamics of (2) resulting in a hybrid VHC of degree $N-1$ for the entire hybrid system (2).

Proposition IV.2. Let $h: \mathbb{R}^{N} \rightarrow \mathbb{R}^{N-1}$ be a VHC of the form (10) for the continuous dynamics of the hybrid EulerLagrange system (2). Then, $h$ is hVHC for (2) if and only if, for all $x \in \mathcal{S} \cap \Gamma$,

$$
p_{i}\left(J_{i}(x)\right)=J_{i+1}(x), i \in\{1, \ldots, N-1\}
$$

and,

$$
\left.\mathrm{d} p_{i}\right|_{x_{1}^{+}} J_{N+1}(x)=J_{N+i+1}(x), i \in\{1, \ldots, N-1\}
$$

where $J_{i}$ is the $i^{\text {th }}$ row of the function $J$ in (2).

Proof. Since $h$ is a VHC, if the system is initialized on the constraint manifold, it remains on it until the solution reaches $x \in \mathcal{S} \cap \Gamma$.

Necessity. Assume that $h$ is a hVHC and suppose $x \in$ $\mathcal{S} \cap \Gamma$. Then $h_{1}(x)=x_{2}-p_{1}\left(x_{1}\right)=0$. But since $h$ is a hVHC in the sense of Definition IV.1 (by hypothesis) $h_{1}\left(x^{+}\right)=$ $x_{2}^{+}-p_{1}\left(x_{1}^{+}\right)=0$. By the definition of $J, x_{2}^{+}=p_{1}\left(x_{1}^{+}\right)$ implies

$$
p_{1}\left(J_{1}(x)\right)=J_{2}(x) .
$$

Similarly, for $x \in \mathcal{S} \cap \Gamma, p_{i}\left(J_{i}(x)\right)=J_{i+1}(x), i \in$ $\{2, \ldots, N-1\}$. This shows that (11) holds.

For $x \in \mathcal{S} \cap \Gamma$ we have $\dot{h}_{1}(x)=x_{N+2}-\left.\mathrm{d} p_{1}\right|_{x_{1}} x_{N+1}=0$. Utilizing the hypothesis and Definition IV.1, we can write

$$
\begin{aligned}
& \dot{h}_{1}\left(x^{+}\right)=x_{N+2}^{+}-\left.\mathrm{d} p_{1}\right|_{x_{1}^{+}}\left(x_{N+1}^{+}\right)=0 \\
& \left.\Rightarrow \mathrm{d} p_{1}\right|_{x_{1}^{+}} x_{N+1}^{+}=x_{N+2}^{+} .
\end{aligned}
$$

By the definition of $J$, the above equation can be written as

$$
\left.\mathrm{d} p_{1}\right|_{x_{1}^{+}} J_{N+1}(x)=J_{N+2}(x) .
$$

Similar arguments shows that, for $x \in \mathcal{S} \cap \Gamma$, $\left.\mathrm{d} p_{i}\right|_{x_{1}^{+}} J_{N+1}(x)=J_{N+i+1}(x)$ for $i \in\{2, \ldots, N-1\}$ which shows that (12) holds.

Sufficiency. Assume that $h$ satisfies (11) and (12). Suppose $x \in \mathcal{S} \cap \Gamma$, then

$$
h_{1}(x)=x_{2}-p_{1}\left(x_{1}\right)=0 .
$$


Since $h$ satisfies (11), we have for $x \in \mathcal{S} \cap \Gamma$

$$
\begin{aligned}
p_{1}\left(J_{1}(x)\right) & =J_{2}(x) \\
\Rightarrow J_{2}(x)-p_{1}\left(J_{1}(x)\right) & =0 \\
\Rightarrow x_{2}^{+}-p_{1}\left(x_{1}^{+}\right) & =0 \\
\Rightarrow h_{1}\left(x^{+}\right) & =0
\end{aligned}
$$

Similarly, $h_{2}\left(x^{+}\right)=h_{3}\left(x^{+}\right)=\cdots=h_{N-1}\left(x^{+}\right)=0$. Now, for $x \in \mathcal{S} \cap \Gamma$, we have $\dot{h}_{1}(x)=x_{N+1}-\left.\mathrm{d} p_{1}\right|_{x_{1}} x_{N+1}=0$. Since $h$ satisfies (12), we have

$$
\begin{aligned}
J_{N+2}(x)-\left.\mathrm{d} p_{1}\right|_{x_{1}^{+}} J_{N+1}(x) & =0 \\
\Rightarrow x_{N+2}^{+}-\left.\mathrm{d} p_{1}\right|_{x_{1}^{+}} x_{N+1}^{+} & =0 \\
\Rightarrow \dot{h}_{1}\left(x^{+}\right) & =0
\end{aligned}
$$

Similarly, $\dot{h}_{i}\left(x^{+}\right)=0, i \in\{2, \ldots, N-1\}$.

The above result allows us to design hVHCs for EulerLagrange systems with impacts (2). We will use this result next in order to design a hVHC for the 2-DOF bipedal robot.

\section{A. hVHC for 2-D.O.F. Bipedal Robot}

Let $h: \mathbb{R}^{2} \rightarrow \mathbb{R}^{1}$ be a VHC of the form (10) for the continuous dynamics of the 2-DOF bipedal robot. Then, by Proposition IV.2, $h$ is hVHC for (9) if and only if, for all $x \in \mathcal{S} \cap \Gamma$,

i) $p \circ p\left(x_{1}\right)=x_{1}$,

ii) $\left.\left.\mathrm{d} p\right|_{x_{1}^{+}}\left(\hat{\mathbf{J}}_{11}+\hat{\mathbf{J}}_{12} \mathrm{~d} p\right)\right|_{x} x_{3}=\left.\left(\hat{\mathbf{J}}_{21}+\hat{\mathbf{J}}_{22} \mathrm{~d} p\right)\right|_{x} x_{3}$.

For the 2-DOF bipedal robot, the constraint equations (10) reduce to

$$
h(x)=x_{2}-p\left(x_{1}\right)
$$

The function $p$ can be any smooth function but, in practice, it is convenient to use constant coefficient polynomials. The next subsection proposes a design procedure that generates a polynomial $p$ satisfying (13) for a 2-DOF bipedal robot.

\section{B. Polynomial Design Generating an hVHC}

We introduce a design procedure that returns a constantcoefficients "shape" polynomial $p$ in (14) satisfying conditions in (13). Let the polynomial be of degree $m \geqslant 3$ written as

$$
p\left(x_{1}\right)=\alpha_{m} x_{1}^{m}+\alpha_{m-1} x_{1}^{m-1}+\cdots+\alpha_{0}, \alpha_{i} \in \mathbb{R} .
$$

The design proceeds as follows.

1) Given a slope $\gamma$, pick a value for the angle of the stance leg just before impact, i.e. $x_{1}^{-}$. For a reasonable gait, $x_{1}^{-} \in(-\pi / 2+\gamma, 0)$. Since the impact results in a new stance angle, $x_{1}^{+}$, we need to set $x_{1}^{+}$accordingly. By (8), $x_{2}^{-}=x_{1}^{+}$. In addition, the impact surface (6) yields $x_{2}^{-}=-x_{1}^{-}-2 \gamma$. Therefore, set $x_{1}^{+}=-x_{1}^{-}-2 \gamma$.

2) We need $x \in \mathcal{S} \cap \Gamma$ just before an impact event. This means that

$$
\begin{aligned}
h\left(x^{-}\right) & =0 \\
\Rightarrow x_{2}^{-}-p\left(x_{1}^{-}\right) & =0 \\
\Rightarrow p\left(x_{1}^{-}\right) & =x_{2}^{-} \\
\Rightarrow p\left(x_{1}^{-}\right) & =x_{1}^{+} \quad(\text { by definition of } J)
\end{aligned}
$$

Therefore, set $p\left(x_{1}^{-}\right)=x_{1}^{+}$. This implies

$$
\sum_{i=0}^{m} \alpha_{i}\left(x_{1}^{-}\right)^{i}=x_{1}^{+} \text {. }
$$

Steps (1) and (2) ensure that condition (i) in (13) holds.

3) We also need that the states remain in $\Gamma$ just after impact which yields the design constraint

$$
\begin{aligned}
h\left(x^{+}\right)=0 & \Rightarrow x_{2}^{+}-p\left(x_{1}^{+}\right)=0 \\
& \Rightarrow p\left(x_{1}^{+}\right)=x_{2}^{+} \\
& \left.\Rightarrow p\left(x_{1}^{+}\right)=x_{1}^{-} \quad \text { (by definition of } J\right) .
\end{aligned}
$$

Therefore, set $p\left(x_{1}^{+}\right)=x_{1}^{-}$to obtain

$$
\sum_{i=0}^{m} \alpha_{i}\left(x_{1}^{+}\right)^{i}=x_{1}^{-} \text {. }
$$

4) Set $\left.\mathrm{d} p\right|_{x_{1}^{-}}=k_{1}$, where $k_{1}$ is a design parameter that determines the change in $x_{1}$ relative to $x_{2}$ just before the impact point. This implies

$$
\sum_{i=1}^{m} i \alpha_{i}\left(x_{1}^{-}\right)^{i-1}=k_{1} .
$$

5) Using the result from Step 4, condition (ii) in (13) implies that

$$
\left.\mathrm{d} p\right|_{x_{1}^{+}}=\frac{\hat{\mathbf{J}}_{21}+\hat{\mathbf{J}}_{22} k_{1}}{\hat{\mathbf{J}}_{11}+\hat{\mathbf{J}}_{12} k_{1}}=: k_{2}
$$

where $\hat{\mathbf{J}}_{n m}, n, m \in\{1,2\}$, are the entries of $\hat{\mathbf{J}}$ defined in (8). We have

$$
\sum_{i=1}^{m} i \alpha_{i}\left(x_{1}^{+}\right)^{i-1}=k_{2} .
$$

In Step 4, the designer must avoid choosing values of $k_{1}$ that result in $\hat{\mathbf{J}}_{11}+\hat{\mathbf{J}}_{12} k_{1}=0$.

6) In matrix form, solve the above four algebraic equations (15)-(18)

$$
X\left[\begin{array}{c}
\alpha_{m} \\
\alpha_{m-1} \\
\vdots \\
\alpha_{0}
\end{array}\right]=\left[\begin{array}{c}
x_{1}^{+} \\
x_{1}^{-} \\
k_{1} \\
k_{2}
\end{array}\right]
$$

for $\alpha_{m}, \ldots, \alpha_{0}$, where the matrix $X \in \mathbb{R}^{4 \times(m+1)}$ is

$$
X:=\left[\begin{array}{ccccc}
x_{1}^{-m} & x_{1}^{-^{m-1}} & \ldots & x_{1}^{-} & 1 \\
x_{1}^{+^{m}} & x_{1}^{+^{m-1}} & \ldots & x_{1}^{+} & 1 \\
m x_{1}^{-m-1} & (m-1) x_{1}^{-m-2} & \ldots & 1 & 0 \\
m x_{1}^{++^{m-1}} & (m-1) x_{1}^{+^{m-2}} & \ldots & 1 & 0
\end{array}\right] .
$$

Proposition IV.3. For $m \geq 3$, system (19) is solvable if $x_{1}^{-} \neq x_{1}^{+}$.

Proof. The $4 \times 4$ submatrix from the last 4 columns of $X$ is

$$
\left[\begin{array}{cccc}
x_{1}^{-3} & x_{1}^{-{ }^{2}} & x_{1}^{-} & 1 \\
x_{1}^{+^{3}} & x_{1}^{+^{2}} & x_{1}^{+} & 1 \\
3 x_{1}^{-^{2}} & 2 x_{1}^{-} & 1 & 0 \\
3 x_{1}^{+^{2}} & 2 x_{1}^{+} & 1 & 0
\end{array}\right]
$$


The determinant of this minor $-\left(x_{1}^{-}-x_{1}^{+}\right)^{4}$ is non-zero since $x_{1}^{-} \neq x_{1}^{+}$which shows that $X$ is onto and (19) is solvable.

The above design procedure ensures the condition $x_{1}^{-} \neq$ $x_{1}^{+}$holds true (Step 1) which naturally leads to feasible constant coefficient polynomials. For the case where $m>3$, there exists infinitely many solutions to (19), which leads to a wide range of possible gaits. As an example, the designer could pick an appropriate solution based on desired rate of change of the stance leg relative to the swing leg, i.e. $\mathrm{d} p\left(x_{1}\right)$.

Next, we discuss more cases where the proposed design procedure is not applicable.

Case $m=2$. In this case, the above design procedure is not applicable since there are 3 unknowns and 4 equations. Therefore, the designer cannot choose $k_{1}$. Instead, Equations (17) and (18) combine into one non-linear equation in the parameters, which needs to be solved simultaneously with (15) and (16).

Case $m=1$. Finally, the conditions (13) cannot be fulfilled since $m=1$ would imply that $k_{1}=k_{2}=\alpha_{1}$, which conflicts the reset map, $J$, of the system.

\section{Control Design}

Introduce a global state transformation $\left(\xi_{1}, \xi_{2}, \eta_{1}, \eta_{2}\right)=$ $T(x):=\left(x_{2}-p\left(x_{1}\right), x_{4}-\mathrm{d} p_{x_{1}} x_{3}, x_{1}, x_{3}\right)$. Let $\xi:=\left(\xi_{1}, \xi_{2}\right)$ and $\eta:=\left(\eta_{1}, \eta_{2}\right)$. Equation (9) becomes,

$$
\begin{array}{ll}
(\dot{\xi}, \dot{\eta})=\hat{f}(\xi, \eta)+\hat{g}(\xi, \eta) \tau & \text { if }(\xi, \eta) \notin \hat{\mathcal{S}} \\
\left(\xi^{+}, \eta^{+}\right)=\hat{J}(\xi, \eta) & \text { if }(\xi, \eta) \in \hat{\mathcal{S}}
\end{array}
$$

where: $\hat{f}=\left.\frac{\partial T}{\partial x} f(x)\right|_{x=T^{-1}(\xi, \eta)}, \hat{g}_{i}=\left.\frac{\partial T}{\partial x} g_{i}(x)\right|_{x=T^{-1}(\xi, \eta)}$, $i \in\{1,2\}, \hat{J}(\xi, \eta):=\left.T(J(x))\right|_{x=T^{-1}(\xi, \eta)}, \hat{\mathcal{S}}=T(\mathcal{S})=$ $\left\{(\xi, \eta) \in \mathbb{R}^{4}: \xi_{1}=-p\left(\eta_{1}\right)-\eta_{1}-2 \gamma\right\}^{3}$.

Throughout the rest of this paper, we refer to $\xi_{1}$ and $\xi_{2}$ dynamics as " $\xi$-dynamics" and to to $\eta_{1}$ and $\eta_{2}$ dynamics as " $\eta$-dynamics". In these new coordinates, the relation $h=$ $x_{2}-p\left(x_{1}\right)$ is enforced by nulling $\xi$-dynamics, which serves as the main control objective. In other words, the hVHC to be enforced in the $(\xi, \eta)$-coordinates reads, $\hat{h}=\xi_{1}$ and the constraint manifold becomes $\hat{\Gamma}:=\left\{(\xi, \eta) \in \mathbb{R}^{4}: \xi_{1}=\right.$ 0 and $\left.\xi_{2}=0\right\}$.

\section{A. Forced Constraint Manifold Dynamics}

Consider the feedback-linearizing control input

$$
\tau=\left.A_{1}^{-1}(x)\right|_{x=T^{-1}(\xi, \eta)}\left[\begin{array}{l}
-\left.L_{f}^{2} h_{1}(x)\right|_{x=T^{-1}(\xi, \eta)}+v_{1} \\
-\left.L_{f}^{2} h_{2}(x)\right|_{x=T^{-1}(\xi, \eta)}+v_{2}
\end{array}\right]
$$

where $h_{1}(x):=x_{2}-p\left(x_{1}\right)$ and $h_{2}(x):=x_{1}, v_{1}$ and $v_{2}$ are two auxiliary control inputs, and the $(i, j)^{\text {th }}$ entry of $A_{1}(x) \in$ $\mathbb{R}^{2 \times 2}$ is $L_{g_{j}} L_{f} h_{i}(x)$. Under the feedback (22), system (21) becomes

$$
\begin{array}{ccc}
\dot{\xi}_{1}=\xi_{2} & \dot{\eta}_{1}=\eta_{2} & \text { if }(\xi, \eta) \notin \hat{\mathcal{S}} \\
\dot{\xi}_{2}=v_{1} & \dot{\eta}_{2}=v_{2} & \\
\left(\xi^{+}, \eta^{+}\right)=\hat{J}(\xi, \eta) & \text { if }(\xi, \eta) \in \hat{\mathcal{S}}
\end{array}
$$

${ }^{3} g_{i}$ is the $i^{\text {th }}$ column of $g$ in (9) and $\hat{g}_{i}$ is the $i^{\text {th }}$ column of $\hat{g}$ in (21). resulting in decoupled $\xi$ and $\eta$ dynamics. The auxiliary input $v_{1}$ is used to null the $\xi$-dynamics whereas the auxiliary input $v_{2}$ is used to enforce tracking of the $\eta_{1}$ state to some desired signal, $\eta_{1}^{d}(\mathrm{t})$. Both auxiliary inputs have a PD structure with gains $K_{P_{i}}$ and $K_{D_{i}}(i \in\{1,2\})$ given as, $v_{1}=-K_{P_{1}} \xi_{1}-$ $K_{D 1} \xi_{2}, v_{2}=-K_{P_{2}} e_{\eta}-K_{D_{2}} \dot{e}_{\eta}+\ddot{\eta}_{1}^{d}$ where $e_{\eta}:=\eta_{1}-\eta_{1}^{d}$.

We simulate (23) for two types of constraints; a VHC and $\mathrm{hVHC}$. The hVHC was generated using the design procedure of Section IV-B.

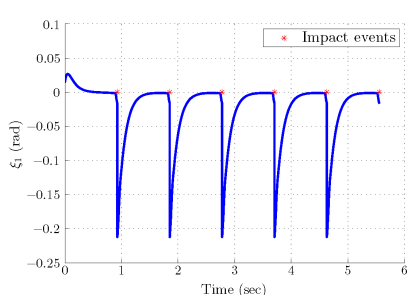

(a) Regulation of the state $\xi_{1}$ using a VHC.

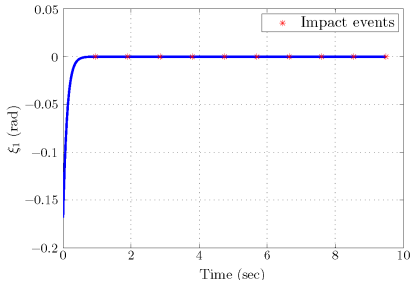

(b) Regulation of the state $\xi_{1}$ using a hVHC.
Fig. 2: The regulation of $\xi_{1}$ using a VHC-based controller and a hVHC-based controller.

In Fig. $2 \mathrm{a}, \xi_{1}$ is regulated during the continuous phase but jumps off-zero at ground impact points. Fig. $2 b$ shows that by using a hVHC the jumps in $\xi_{1}$ at ground impact points are eliminated.

Fig. 3a shows the resulting gait shape in $x$-coordinates. The designer can easily generate other gait shapes by using different solutions to (19). Fig. 3b illustrates the motion of one of the robot's legs, say leg 2, while performing the gait where the angular position of leg 2 is denoted by $\theta_{2}$.

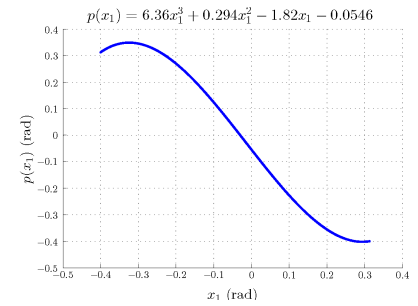

(a) Desired gait shape is captured by the polynomial $p$.

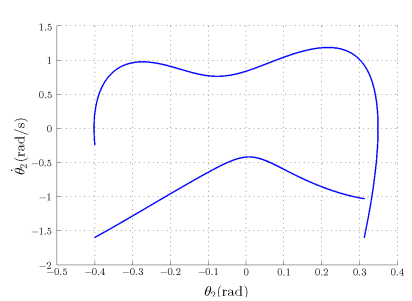

(b) The motion of leg 2 generated using the polynomial $p$.
Fig. 3: Gait shape defined by a hVHC.

\section{B. Unforced Constraint Manifold Dynamics}

This section discusses hVHC enforcement while leaving the dynamics on the constraint manifold, the $\eta$-dynamics, unforced. Consider the control law

$$
\tau=\left[A_{2}^{+}(x)\left(v-L_{f}^{2} h_{1}(x)\right)\right]_{x=T^{-1}(\xi, \eta)}
$$

where $v$ is a auxiliary control input, $A_{2}(x)$ := $\left[L_{g_{1}} L_{f} h_{1}(x) \quad L_{g_{2}} L_{f} h_{1}(x)\right]$, and $A_{2}^{+}$is a pseudo-inverse of $A_{2}$. Then, system (21) becomes

$$
\begin{array}{ccc}
\dot{\xi}_{1}=\xi_{2} & \dot{\eta}_{1}=\eta_{2} & \text { if }(\xi, \eta) \notin \hat{\mathcal{S}} \\
\dot{\xi}_{2}=v & \dot{\eta}_{2}=\nu(\xi, \eta) & \\
\left(\xi^{+}, \eta^{+}\right)=\hat{J}(\xi, \eta) & \text { if }(\xi, \eta) \in \hat{\mathcal{S}}
\end{array}
$$


where $\nu(\eta, \xi):=L_{f}^{2} h_{2}(x)_{x=T^{-1}(\xi, \eta)}+$

$\left.\left[L_{g_{1}} L_{f} h_{2}(x), L_{g_{2}} L_{f} h_{2}(x)\right]\left(A_{2}^{+}\left(v-L_{f}^{2} h_{1}(x)\right)\right)\right|_{x=T^{-1}(\xi, \eta)}$

The $\xi$-dynamics can be regulated by a PD auxiliary controller,

$$
v=-K_{P} \xi_{1}-K_{D} \xi_{2}
$$

Again, this controller was tested numerically using VHCs and hVHCs separately with the results similar to those shown in Fig. 2.

Numerical simulations suggest that the unforced dynamics under the constraint manifold are stable. On the constraint manifold, the convergence of these dynamics strongly depend on the initial conditions. Solutions either converge to the desired gait or they converge to a stable fixed point, where the robot enters a stand-still state. Fig. 4 categorizes the initial conditions into 3 distinct regions and summarizes four different behaviors.

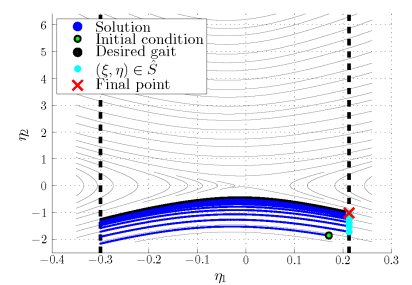

(a) Region 1: convergence to the gait.

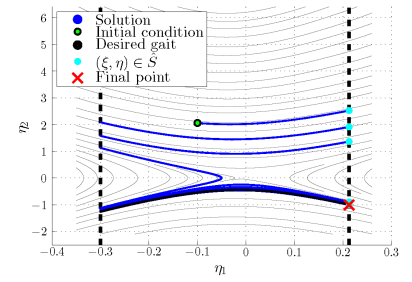

(c) Region 3(a): convergence to the gait.

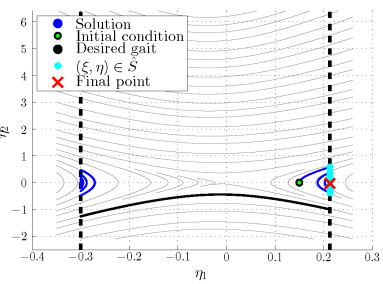

(b) Region 2: convergence to a fixed-point.

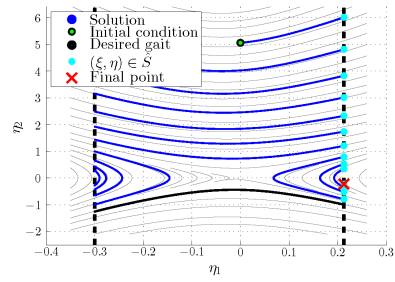

(d) Region 3(b): convergence to a fixed-point.

Fig. 4: Dynamics on $\hat{\Gamma}$ with different initial conditions.

\section{CONCLUSIONS}

This paper applied the notion of virtual holonomic constraints to Euler-Lagrange systems with impacts. For a 2DOF bipedal robot, a design procedure was developed that returns a feasible hVHC. Two control laws that enforce a given hVHC were designed and numerically tested. The first design controls the dynamics on the constraint manifold. The second controller left the dynamics on the constraint manifold unforced. The unforced dynamics were shown, through simulations, to be stable. The investigation of robustness to modelling uncertainty and proof of stability for the uncontrolled contraint manifold dynamics are subjects of future research.

\section{REFERENCES}

[1] A. Shiriaev, J. W. Perram, and C. Canudas-de Wit, "Constructive tool for orbital stabilization of underactuated nonlinear systems: Virtual constraints approach," IEEE Transactions on Automatic Control, vol. 50, no. 8, pp. 1164-1176, 2005.

[2] L. Freidovich, A. Shiriaev, and I. Manchester, "Stability analysis and control design for an underactuated walking robot via computation of a transverse linearization," in Proceedings of the 17th IFAC World Congress, Seoul, Korea, 2008, pp. $10166-10171$.

[3] M. Maggiore and L. Consolini, "Virtual holonomic constraints for Euler-Lagrange systems," IEEE Transactions on Automatic Control, vol. 58, no. 4, pp. 1001-1008, 2013.

[4] M. Vukobratović and B. Borovac, "Zero-moment point: thirty five years of its life," International Journal of Humanoid Robotics, vol. 1, no. 1, pp. 157-173, 2004.

[5] Y. Hu and Z. Lin, "Balance control of planar biped robots using virtual holonomic constraints," Robotica, pp. 1-16, 2014.

[6] E. Westervelt, J. Grizzle, and D. Koditschek, "Zero dynamics of underactuated planar biped walkers," in IFAC 15th Triennial World Congress, 2002.

[7] J. Grizzle, G. Abba, and F. Plestan, "Asymptotically stable walking for biped robots: Analysis via systems with impulse effects," IEEE Transactions on Automatic Control, vol. 46, no. 1, pp. 51-64, 2001.

[8] E. R. Westervelt, J. W. Grizzle, and D. E. Koditschek, "Hybrid zero dynamics of planar biped walkers," IEEE Transactions on Automatic Control, vol. 48, no. 1, pp. 42-56, 2003.

[9] A. D. Ames, K. Galloway, K. Sreenath, and J. W. Grizzle, "Rapidly exponentially stabilizing control Lyapunov functions and hybrid zero dynamics," IEEE Transactions on Automatic Control, vol. 59, no. 4, pp. 876-891, 2014.

[10] B. Morris and J. W. Grizzle, "Hybrid invariant manifolds in systems with impulse effects with application to periodic locomotion in bipedal robots," IEEE Transactions on Automatic Control, vol. 54, no. 8, pp. 1751-1764, 2009.

[11] K. Sreenath, H.-W. Park, I. Poulakakis, and J. W. Grizzle, "A compliant hybrid zero dynamics controller for stable, efficient and fast bipedal walking on MABEL," The International Journal of Robotics Research, vol. 30, no. 9, pp. 1170-1193, 2011.

[12] H.-W. Park, A. Ramezani, and J. Grizzle, "A finite-state machine for accommodating unexpected large ground-height variations in bipedal robot walking," IEEE Transactions on Robotics, vol. 29, no. 2, pp. 331-345, 2013.

[13] K. Sreenath, H.-W. Park, I. Poulakakis, and J. Grizzle, "Embedding active force control within the compliant hybrid zero dynamics to achieve stable, fast running on MABEL," The International Journal of Robotics Research, vol. 32, no. 3, pp. 324-345, 2013.

[14] G. Yan, C. Tang, Z. Lin, and I. Malloci, "Feedback control for compass-like biped robot with underactuated ankles using transverse coordinate transformation," Robotica, pp. 1-15, 2014.

[15] A. S. Shiriaev and L. B. Freidovich, "Transverse linearization for impulsive mechanical systems with one passive link," IEEE Transactions on Automatic Control, vol. 54, no. 12, pp. 2882-2888, 2009.

[16] F. Asano, M. Hashimoto, N. Kamamichi, and M. Yamakita, "Extended virtual passive dynamic walking and virtual passivity-mimicking control laws," in IEEE International Conference on Robotics and Automation, vol. 3. IEEE, 2001, pp. 3139-3144.

[17] F. Asano, M. Yamakita, N. Kamamichi, and Z. Luo, "A novel gait generation for biped walking robots based on mechanical energy constraint," IEEE Transactions on Robotics and Automation, vol. 20, no. 3, pp. 565-573, 2004.

[18] M. A. Lawati, "Gait shape control for 2-D.O.F. bipedal robots using hybrid virtual holonomic constraints," Master's thesis, University of Waterloo, 2014.

[19] M. W. Spong, J. K. Holm, and D. Lee, "Passivity-based control of bipedal locomotion," IEEE Robotics \& Automation Magazine, vol. 14, no. 2, pp. 30-40, 2007.

[20] M. Garcia, A. Chatterjee, A. Ruina, M. Coleman et al., "The simplest walking model: Stability, complexity, and scaling," Journal of Biomechanical Engineering, vol. 120, no. 2, pp. 281-288, 1998.

[21] R. Goebel, R. Sanfelice, and A. Teel, "Hybrid dynamical systems," IEEE Control Systems Magazine, vol. 29, no. 2, pp. 28-93, 2009. 